\title{
Wavelet Neural Network Methodology for Ground Resistance Estimation
}

\author{
Vasilios P. Androvitsaneas ${ }^{* a}$, Antonios K. Alexandridis ${ }^{b}$, Ioannis F. Gonos ${ }^{a}$, \\ Georgios D. Dounias ${ }^{c}$, Ioannis A. Stathopulos ${ }^{a}$ \\ ${ }^{\text {a }}$ High Voltage Laboratory, School of Electrical and Computer Engineering, National \\ Technical University of Athens, 9 Iroon Politechniou Street, Zografou Campus, Athens \\ 15780, Greece \\ e-mail: v.andro@mail.ntua.gr ${ }^{*}$,igonos@cs.ntua.gr, stathop@power.ece.ntua.gr \\ ${ }^{\mathrm{b}}$ School of Mathematics, Statistics and Actuarial Science, University of Kent, Cornwallis \\ Building, P.O. CT2 7NF, Canterbury, UK \\ e-mail: A.Alexandridis@kent.ac.uk \\ ${ }^{\mathrm{c}}$ Management \& Decision Engineering Lab, Department of Financial and Management \\ Engineering, University of the Aegean, 41 Kountouriotou Street, 82100 Chios, Greece \\ e-mail: g.dounias@aegean.gr
}

\begin{abstract}
The present study aims to develop an estimation model for the ground resistance fluctuation. The need of engineers to have in their disposal a flexible and reliable tool, for estimating and predicting grounding systems behavior, is what actually motivated this workscope. It is well-known that grounding systems are a key of high importance for the safe operation of electrical facilities, substations, transmission lines and, generally, electric power systems. Yet, in most cases, electrical engineers and researchers have few data about the soil resistivity variation at the terrain of interest in design phase and, even more, the periodic measurement of ground resistance is hindered very often by the residence and building infrastructure, after the installation. Thus, the proposed model aspires to offer a reliable solution to estimation problems of
\end{abstract}

${ }^{*}$ Corresponding author 
ground resistance. It consists of a Wavelet Neural Network (WNN), which has been trained by field measurements of soil resistivity and rainfall height, gathered/observed the last four years. Grounding rods encased in ground enhancing compounds and in natural soil have been tested, so that a wide dataset for the training of the network can be obtained, covering various soil conditions. Furthermore, the proposed estimation model can be used for the estimation of the behavior of several ground enhancing compounds, frequently used in grounding practice. The nature of this problem and the data structure favor the proposed WNN methodology, due to high accuracy and performance it presents in solving such problems. Therefore, this paper introduces the wavelet analysis in the field of ground resistance estimation and endeavors to take advantage of the benefits of computational intelligence.

Keywords: grounding systems; ground enhancing compounds; ground resistance; wavelet neural networks; forecasting; computational intelligence.

\section{Introduction}

Grounding systems are an integral part of the protection system for electrical facilities and electric power systems against lightning and power frequency fault currents, as they are designed to dissipate high magnitude fault currents into the earth through a safe passage in the shortest possible time. Their purpose is to keep at minimum the ground potential rise (GPR), consequence of a discharging fault current, so as to ensure the safety of people and equipment from electric shock. Nevertheless, the assumption that any grounded object can be safely touched is not always correct. Under fault conditions, the ground potential rise could reach hazardous levels that may well lead to human losses and equipment destruction. Thus, for a well-designed grounding system in order to provide constant and full protection, technical measures 
are necessary to ensure a good and consistent behavior of the system throughout its lifecycle.

As far as the power frequency resistance is concerned, a grounding system must maintain a low resistance in respect to remote earth during its service. In this way, the decline in potential rise can restrain the high values of step and touch voltages in the facility and its vicinity, which are able to jeopardize human lives. International standards [1-3] highlight the variation of ground resistance value under the effect of soil structure and soil moisture. Therefore, for safety reason, regular measurement of grounding systems is recommended, [1-3].

However, in most of the cases, an electrical engineer has to deal with confined spaces for the construction of an effective grounding system, or with the huge cost which often may be inhibitive for the construction. Furthermore, soil resistivity of the upper layer is subjected to seasonal variation due to weather conditions, such as rainfall, ice and air temperature, which mainly effect on soil humidity, while the dissolved salts percentage and the soil structure play a major role in soil resistivity value [4-6]. In the last decades the usage of ground enhancing compounds for soil alleviation and decreasing the ground resistance value becomes more and more popular in engineering field.

Despite the recommendations of the standards, the periodic measurement of ground resistance is hindered very often by the residence and building infrastructure. Moreover, many times it is essential for engineers to have an estimation of the behavior of constructed or, in design phase, grounding systems over time. This work endeavors to develop a novel tool for estimating and forecasting the ground resistance values of several grounding systems, based on soil resistivity measurements at the location of interest and on local rainfall data, using WNN. 


\section{Wavelet Neural Networks}

\subsection{General description}

Wavelet neural networks or, simply wavelet networks (WNs), are a new class of networks that combine the classic sigmoid neural networks (NNs) and the wavelet analysis (WA). WNNs have been used with great success in a wide range of applications. Wavelet analysis has proved to be a valuable tool for analyzing a wide range of time-series and has already been used with success in image processing, signal de-noising, density estimation, signal and image compression and time-scale decomposition. It is often regarded as a "microscope" in mathematics [7] and it is a powerful tool for representing nonlinearities [8]. However, WA is suitable for applications of small input dimension, since the construction of a wavelet basis is computationally expensive when the dimensionality of the input vector is relatively high [9].

Wavelet analysis decomposes a general function or signal into a series of (orthogonal) basis functions called wavelets, which have different frequency and time locations. More precisely, wavelet analysis decomposes time-series and images into component waves of varying durations called wavelets, which are localized variations of a signal $[10,11]$. As illustrated by Donoho and Johnstone [12], the wavelet approach is very flexible in handling very irregular data series. Ramsey [13] also comments that wavelet analysis has the ability to represent highly complex structures without knowing the underlying functional form, which is of great benefit in economic and financial research. A particular feature of the signal analyzed can be identified with the positions of the wavelets into which it is decomposed.

WNNs were proposed by Zhang and Benveniste [14] as an alternative to feedforward neural networks. The wavelet networks are a generalization of radial basis 
function networks. They are one hidden-layer networks that use a wavelet as an activation function, instead of the classic sigmoidal family. It is important to mention here that the multidimensional wavelets preserve the "universal approximation" property that characterizes neural networks. The nodes (or wavelons) of the hidden layer are the wavelet coefficients of the function expansion that have a significant value. In Bernard et al. [15] various reasons were presented explaining why wavelets should be used instead of other transfer functions. In particular, firstly, wavelets have high compression abilities and, secondly, computing the value at a single point or updating the function estimate from a new local measure, involves only a small subset of coefficients.

2.2 Proposed WNN methodology and architecture for the estimation of ground resistance

In this study, a multidimensional WNN with a linear connection between the hidden units (wavelons) and the output is implemented. Moreover, in order for the model to perform well in the presence of linearity, direct connections from the input layer to the output layer are established. The structure of a single hidden-layer feedforward wavelet network is given in Fig. 1. The network output is given by the following expression:

$$
g_{\lambda}(\mathbf{x} ; \mathbf{w})=\hat{y}(\mathbf{x})=w_{\lambda+1}^{[2]}+\sum_{j=1}^{\lambda} w_{j}^{[2]} \cdot \Psi_{j}(\mathbf{x})+\sum_{i=1}^{m} w_{i}^{[0]} \cdot x_{i}
$$

In the above expression, $\Psi_{j}(\mathbf{x})$ is a multidimensional wavelet which is constructed by the product of $m$ scalar wavelets, $\mathbf{x}$ is the input vector, $m$ is the number of network inputs, $\lambda$ is the number of hidden units (HUs) and $w$ stands for a network weight. The multidimensional wavelets are computed as follows:

$$
\Psi_{j}(x)=\prod_{i=1}^{m} \psi\left(z_{i j}\right)
$$


where $\psi$ is the mother wavelet and

$$
z_{i j}=\frac{x_{i}-w_{(\xi) i j}^{[1]}}{w_{(\zeta) i j}^{[1]}}
$$

In the above expression, $i=1, \ldots m, j=1, \ldots \lambda+1$ and the weights $w$ correspond to the translation $\left(w_{(\xi) i j}^{[1]}\right)$ and the dilation $\left(w_{(\zeta) i j}^{[1]}\right)$ factors. The complete vector of the network parameters comprises $w=\left(w_{i}^{[0]}, w_{j}^{[2]}, w_{\lambda+1}^{[2]}, w_{(\xi) i j}^{[1]}, w_{(\zeta) i j}^{[1]}\right)$. These parameters are adjusted during the training phase. Furthermore, the second derivative of the Gaussian, the so-called "Mexican Hat" wavelet is used which proved to be useful and to work satisfactorily in various applications [18-20]:

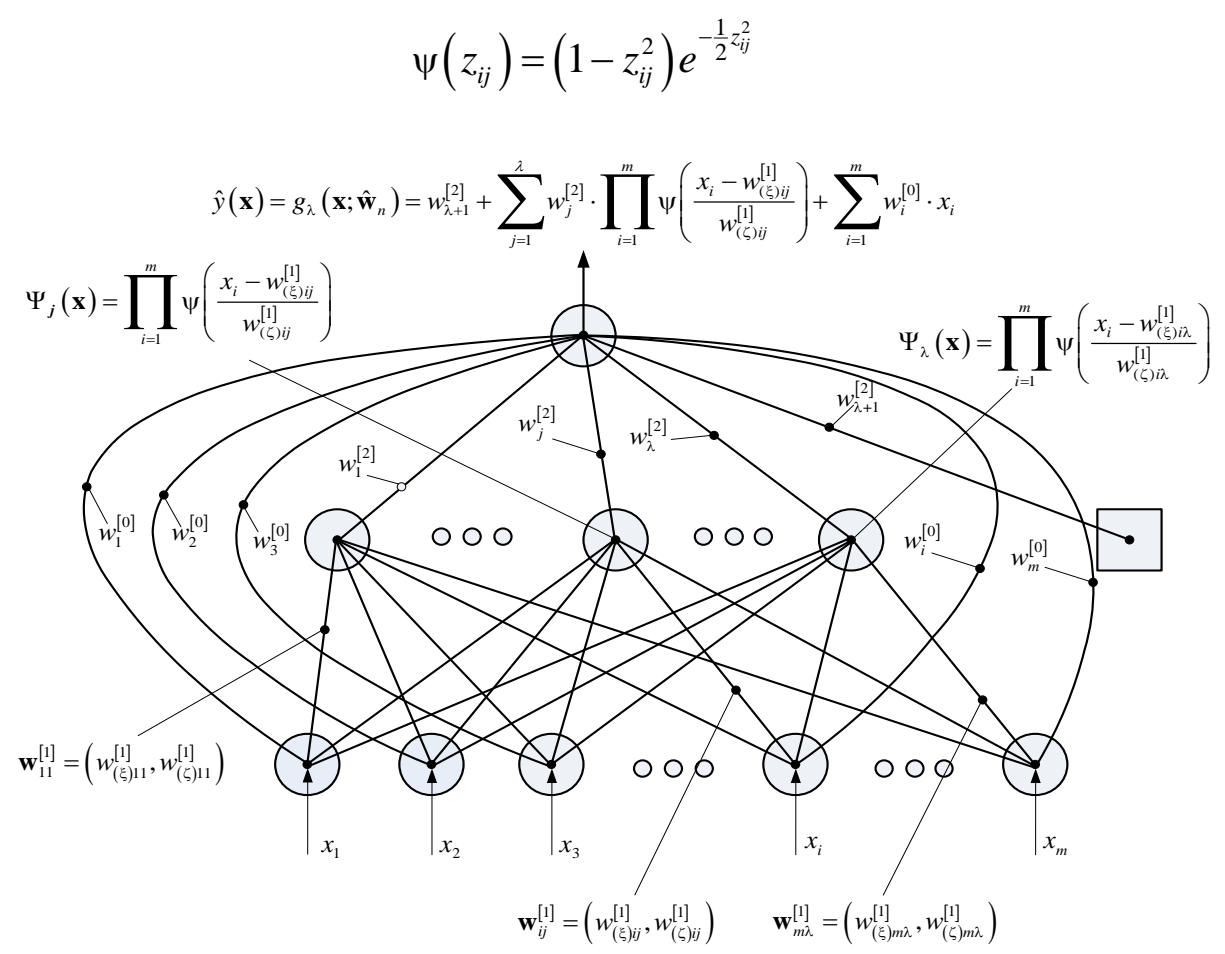

Fig.1

A wavelet is a waveform of effectively limited duration that has an average value of zero and localized properties. Hence, a random initialization may lead to wavelons with a value of zero, affect the speed of training and lead to a local minimum of the loss function. Utilizing the information that can be extracted by the WA from the input 
dataset, the initial values of the parameters $w$ of the network can be selected in an efficient way. Efficient initialization will result in less iterations in the training phase of the network and in training algorithms that will avoid local minima of the loss function in the training phase. In the present network the Backward Elimination (BE) method $[9,20]$ is used for the initialization of the network parameters. The BE starts the regression by selecting all the available wavelets from the wavelet library. Then, the wavelet that contributes the least in the fitting of the training data is repeatedly eliminated. The drawback of $\mathrm{BE}$ is that it is computationally expensive but it is considered to have good efficiency.

After the initialization phase, the network is further trained in order to obtain the vector of the parameters $w=\hat{\mathbf{w}}_{n}$ which minimizes the loss function. The ordinary backpropagation algorithm (BP) is used for the training of the WNN, as it is probably the most popular algorithm used for training of WNNs. BP is less fast but also less prone to sensitivity to initial conditions than higher order alternatives. According to this algorithm the weights of the network are trained to minimize the mean squared error function (or loss function), which is given by the following formula:

$$
L_{n}=\frac{1}{n} \sum_{p=1}^{n} E_{p}=\frac{1}{2 n} \sum_{p=1}^{n} e_{p}^{2}=\frac{1}{2 n} \sum_{p=1}^{n}\left(y_{p}-\hat{y}_{p}\right)^{2}
$$

where $y_{p}$ is the target value, $\hat{y}_{p}$ the network output and $n$ the number of the patterns in the training set.

Thus, the weights $w_{i}^{[0]}, w_{j}^{[2]}$ and the parameters $w_{(\xi) i j}^{[1]}$ and $w_{(\zeta) i j}^{[1]}$ are trained during the learning phase for approximating the target function. A key decision related to the training of a WNN is the time the weight adjustment should end. Under the assumption that the WNN contains the number of wavelets that minimizes the prediction risk, the training stops when one of the following criteria is met: the cost function reaches a fixed 
lower bound, or the variations of the gradient or the variations of the parameters reaches a lower bound. These stopping criteria can be mathematically expressed as:

$$
\begin{gathered}
\left|L_{n}(e p)-L_{n}(e p-1)\right| \leq \text { limit }_{1} \\
\left|\frac{\partial L_{n}(e p)}{\partial w_{t}}-\frac{\partial L_{n}(e p-1)}{\partial w_{t}}\right| \leq \text { limit }_{2}
\end{gathered}
$$

where $L_{n}$ is the loss function, ep is the epoch and limit 1 and limit 2 are the predefined lower bounds.

Afterwards, one of the most crucial steps is to identify the correct topology of the network. A desired WNN architecture should contain as few HUs as necessary while at the same time it should explain as much variability of the training data as possible. The Minimum Prediction Risk (MPR) principle can be applied as the most suitable measure of the generalization ability of the network. The idea behind MPR is to estimate the out-of-sample performance of incrementally growing networks. More precisely, the prediction risk of a network $\mathbf{g}_{\lambda}\left(\mathbf{x} ; \hat{\mathbf{w}}_{n}\right)$ is the expected performance of the network on new data that have not been introduced during the training phase and is given by:

$$
P_{\lambda}=E\left[\frac{1}{n} \sum_{p=1}^{n}\left(y_{p}^{*}-\hat{y}_{p}^{*}\right)^{2}\right]
$$

In order to estimate the prediction risk and to find the network with the best predicting ability, a series of information criteria has developed. In this case, the Bayesian Information Criterion (BIC) is considered to be the most appropriate among the other criteria for the WNN construction, as its little computational burden doesn't affect the precision on estimations. First the WNN is constructed with zero HUs. Then, the corresponding information criterion is estimated. Next, one HU is added to the network and the procedure is repeated until the network contains a predefined maximum number of HUs. The number of HUs that produces the minimum prediction 
risk is the number of the appropriate wavelets for the construction of WNN. The BIC is expressed as:

$$
J_{B I C}=\frac{1}{n} \sum_{p=1}^{n}\left(y_{p}-\hat{y}_{p}\right)^{2}+\frac{k \hat{\sigma}^{2} \ln (n)}{n}
$$

where $k$ is the number of the parameters of the network, $n$ the number of the training patterns and $\sigma^{2}$ the noise variance estimator.

Finally, a variable selection algorithm is applied during the WNN construction, aiming to determine the most significant input variables for the network output. In real problems it is important to determine correctly the explanatory variables. In most problems there is a little information about the relationship of any explanatory variable with the dependent variable. As a result, unnecessary explanatory variables are included in the model reducing its predictive power. Among various sensitivity criteria and model fitness criteria the Sensitivity Based Pruning (SBP) [11] is chosen for the variable selection of the examined architecture. The SBP method quantifies a variable's relevance to the model by the effect on the empirical loss of the replacement of that variable by its mean and is given by:

$$
\operatorname{SBP}\left(x_{j}\right)=L_{n}\left(\mathbf{x} ; \hat{\mathbf{w}}_{n}\right)-L_{n}\left(\overline{\mathbf{x}}^{(j)} ; \hat{\mathbf{w}}_{n}\right)
$$

where

$$
\overline{\mathbf{x}}^{(j)}=\left(x_{1, t}, x_{2, t}, \ldots, \bar{x}_{j}, \ldots, x_{m, t}\right)
$$

and

$$
\bar{x}_{j}=\frac{1}{n} \sum_{t=1}^{n} x_{j, t}
$$

The proposed methodology for the estimation of ground resistance value of each rod can be concisely illustrated in the flowchart of Fig. 2. 


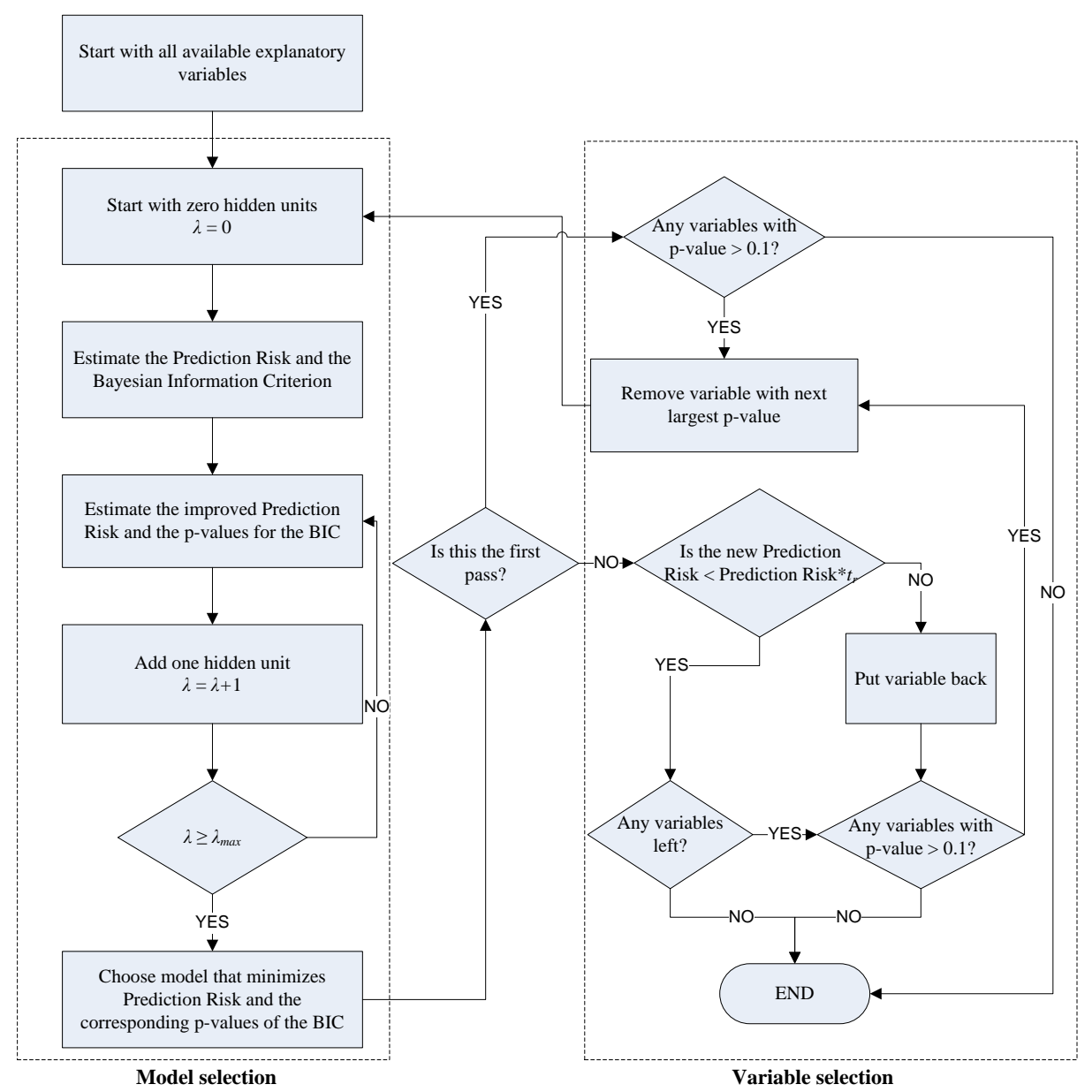

Fig.2 


\section{Experimental settings and results}

\subsection{Experimental set-up and measurements}

The necessary dataset for the training of the developed WN has been obtained from

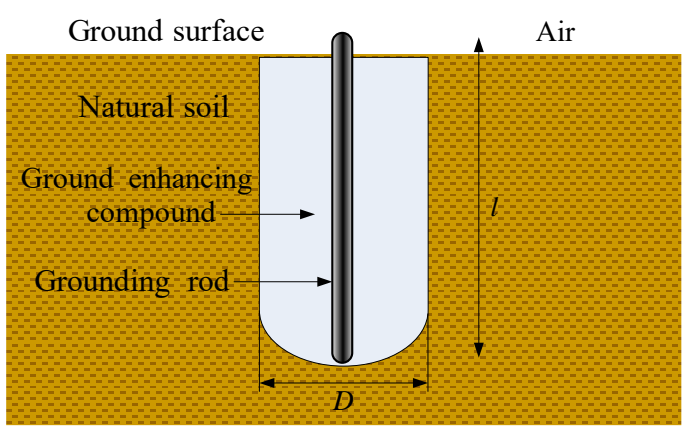
the measurements of a full scale field test. In the present work, five grounding rods, St/e-Cu type A, dimensioned $17 \times 1500 \mathrm{~mm}$, with a minimum copper thickness $254 \mu \mathrm{m}$, have been evaluated in field conditions [16].

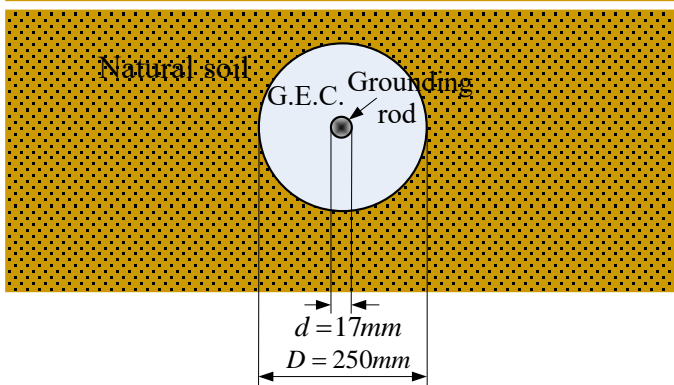

Fig.3
The $\operatorname{rod} \mathrm{G}_{1}$ has been driven into natural soil, while $\mathrm{G}_{2}$ has been encased in conductive concrete, $\mathrm{G}_{3}$ in slurry bentonite, $\mathrm{G}_{4}$ and $\mathrm{G}_{5}$ in commercial chemical ground enhancing compounds, tagged as compound $\mathrm{A}$ and

compound B respectively. A schematic representation of each rod encased in ground enhancing compound for this experiment is given in Fig. 3. The cylinder of Fig. 3 contains a different enhancing compound each time and, therefore, this results in four grounding systems with different behavior and time variation of their ground resistance. The soil in the testing terrain is composed of cobbles and gravel in the percentage of $54.8 \%$, sand in $39.5 \%$ and silt clay in $5.7 \%$.

The measurements performed at the experimental field, for a period of 44 months, concern soil resistivity $(\rho)$ in the depths of $1 \mathrm{~m}, 2 \mathrm{~m}, 4 \mathrm{~m}, 6 \mathrm{~m}$ and $8 \mathrm{~m}$, ground resistance $\left(R_{g}\right)$ of the five tested rods and rainfall height $(r)$. The ground resistance of each rod has been measured using the fall of potential method and the soil resistivity according to the Wenner method. For this purpose, a Megger / DET2/2 auto earth tester has been used. The experimental process is fully complied with the specifications of the relative 
standard [3]. The rainfall data have been collected from the database of a meteorological station which is installed near the testing field, inside the university campus, and it is in the service of the Hydrological Observatory of Athens [17]. The results of the field measurements used for the construction of the WNN estimation model are illustrated in Figs. 4 and 5.

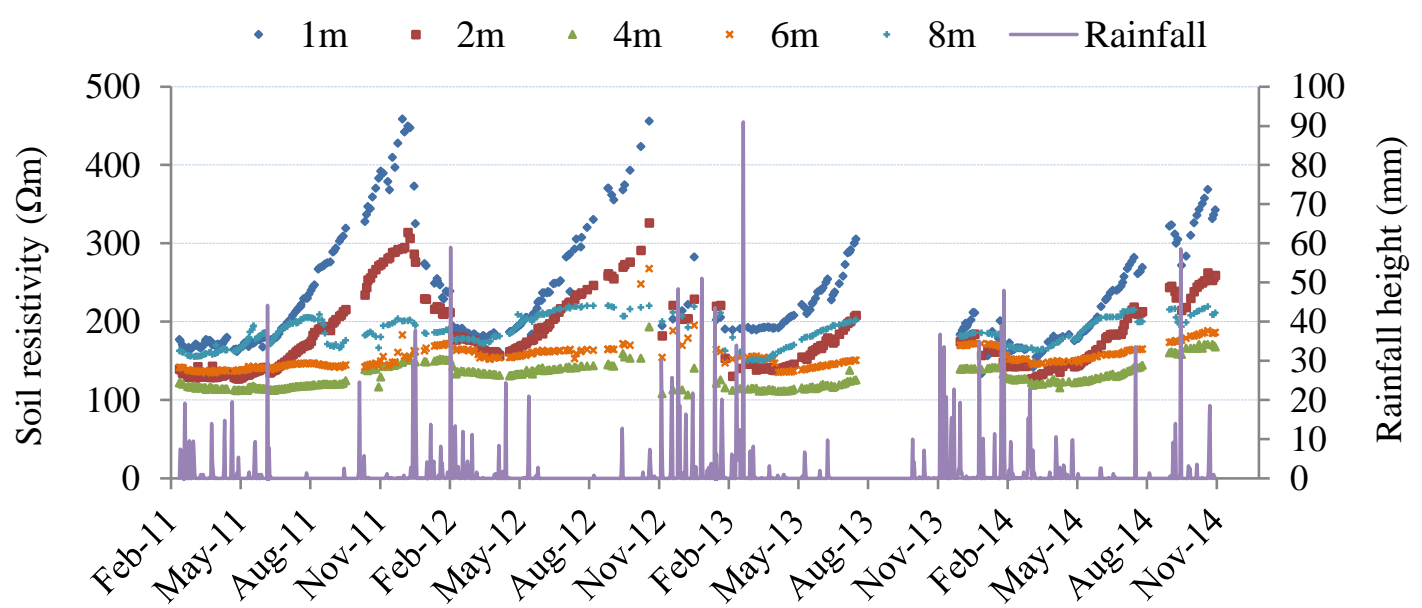

Fig. 4

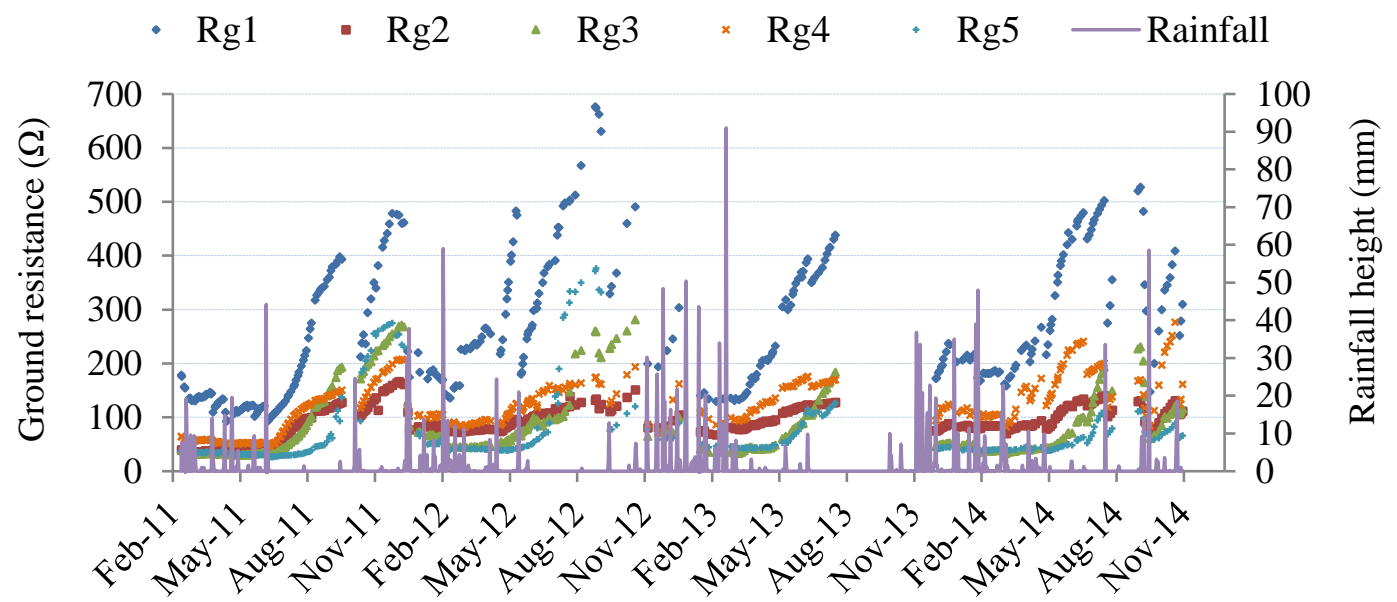

Fig. 5

\subsection{Application of $\mathrm{WNN}$ and results}

In previous work [21], a non-linear non-parametric WNN model has developed using a smaller dataset which contained the measurements from three tested rods of the same type in natural soil, conductive concrete and chemical compound. In the present 
work for the problem of ground resistance estimation a multidimensional WNN with a linear connection between the HUs and the output is applied.

The set of independent variables consists of: a) soil resistivity, $\rho_{i d}$, in $1 \mathrm{~m}, 2 \mathrm{~m}, 4 \mathrm{~m}$, $6 \mathrm{~m}$ and $8 \mathrm{~m}$ depth, $i$, on the day, $d$. (Fig. 4), b) the mean weekly soil resistivity, $\rho_{i w}$, in the same depths which is the average of the last seven days c) the mean monthly soil resistivity, $\rho_{i m}$, in depths of $1 \mathrm{~m}$ and $2 \mathrm{~m}$ which is the average of the last month, d) the total rainfall height of the measurement day, $r_{d}$, e) the weekly total rainfall height, $r_{w}$, which is the total rainfall height in the last seven days and f) the total rainfall height for the last month, $r_{m}$. It is noted that $i=1,2,4,6,8 \mathrm{~m}$ in depth. The dependent or output variable is the ground resistance $R_{g}$ of each tested grounding system the next day (Fig. 5). Hence, we use the measurements that are available today in order to forecast the ground resistance, $R_{g}$, tomorrow. For each rod a separate network is constructed.

The initialization of the network parameters is performed by the BE method, starting the regression by selecting all the available wavelets from the wavelet library. Then, the wavelet that contributes the least in the fitting of the training data is repeatedly eliminated.

The experimental dataset comprises 337 input/output patterns, ranging from February 2011 to November 2014. It is composed of the values illustrated, in detail, in Figs. 4 and 5 and it is split randomly into two sets:

- The training set (or in-sample set) consists of 237 patterns (i.e. $70 \%$ of the original dataset) and is used for the training of the network. In other words, the construction of the architecture of the network, i.e. find the optimal number of hidden units, the variable selection as well as to learn the relationship between the input and the output variables. 
- The forecasting set (or out-of-sample set) consists of 100 patterns (i.e. $30 \%$ of the original dataset) and is used for the evaluation of the predicting ability of the network. Note that the out-of-sample set was not used during the training of the network.

A common approach is to further divide the in-sample set into a training set and a validation set. Instead, one of the advantages of the Model Identification algorithm, [10], outlined in Fig. 2, is that a validation set is not needed. As a result, a better training of the WNN is obtained since the whole available in-sample dataset is used for training. The WNN is trained with the use of the Batch mode with constant learning rate $\eta=0.1$ and zero momentum term. The second derivative of the Gaussian, i.e. the "Mexican Hat" wavelet, given by (4), is used as an activation function. For the model and the variable selection, the BIC and the SBP criteria were used respectively.

In [21], the proposed algorithm has been carried out for two scenarios. In the first one, the variable selection algorithm with the SBP criterion was applied in order to find the statistical significant variables. Hence, only a subset of the available variables were used for the network training. In the second one, no variable selection was applied and all available input variables of the dataset were used. The preliminary results in [21] indicate that, applying the variable selection algorithm (SBP), the network performed much better, yielding very high correlation values. As it is presented in [10-11] the variable selection algorithm can reduce the complexity of the network, reduce training time and improve the network's predicting ability.

Hence, the authors, propelled by the results of the previous study, decided to apply the SBP as a variable selection method for all the electrodes. In Table 1 the selected variables as well as the optimal number of HUs for each network are presented. In addition various error criteria, such as the normalised mean square error (NMSE) and 
the symmetrical mean absolute percentage error as well as the $R^{2}$ and the $\bar{R}^{2}$ are presented.

The fitting results of the out-of-sample set for the five electrodes are illustrated in Figs. 6-10. The horizontal axis is calibrated according to the serial number of the outof-sample patterns. It is clear that, when the variable selection framework is applied, the WNN can accurately approximate the real data.

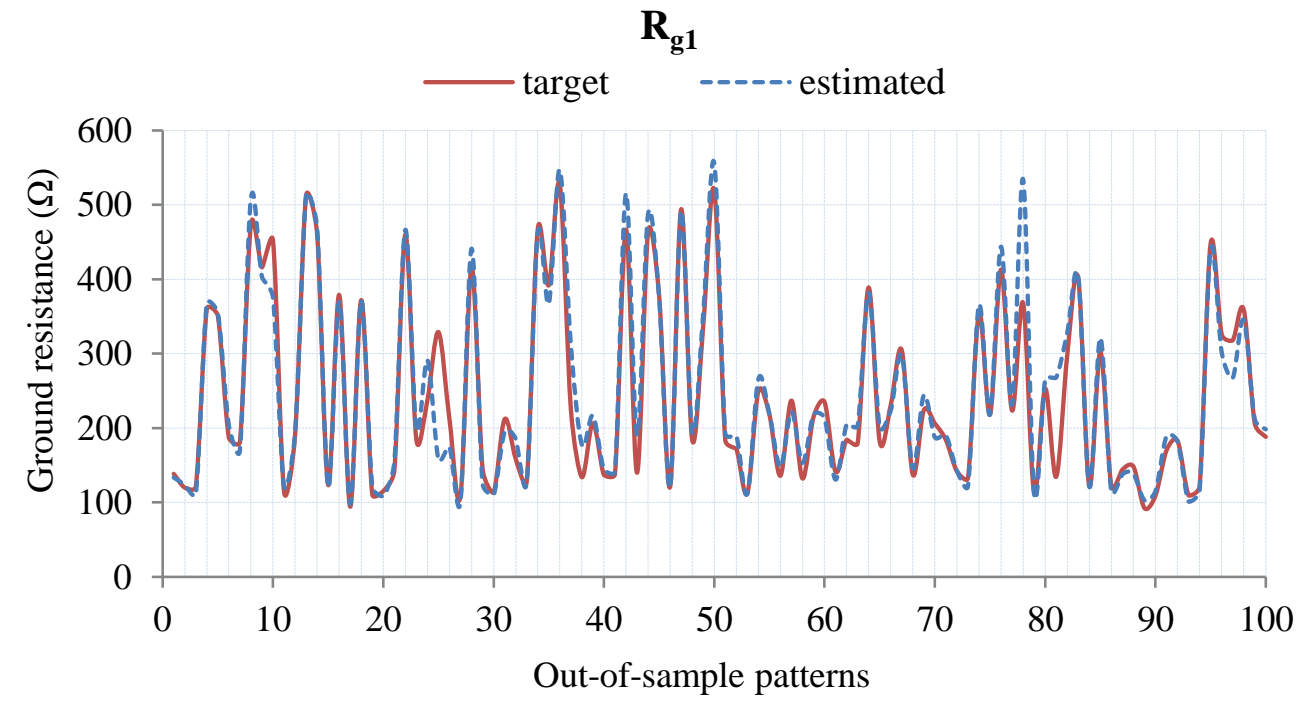

Fig. 6

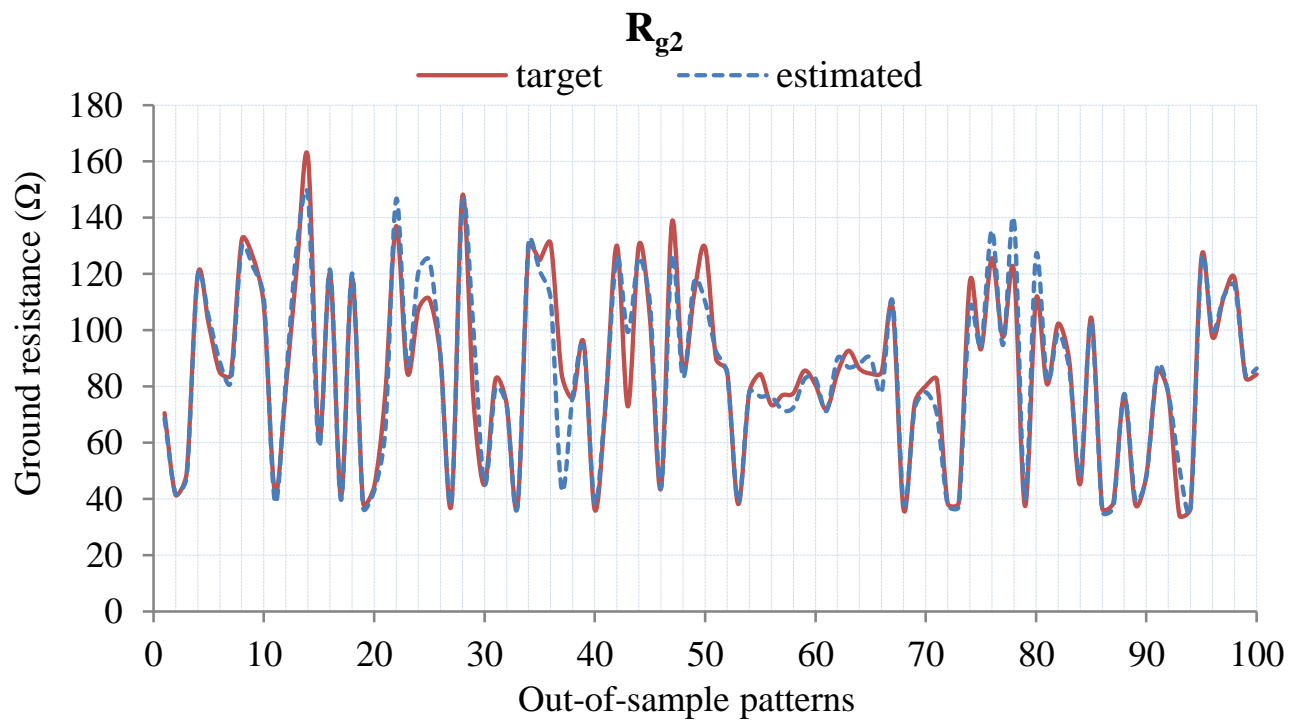

Fig. 7 


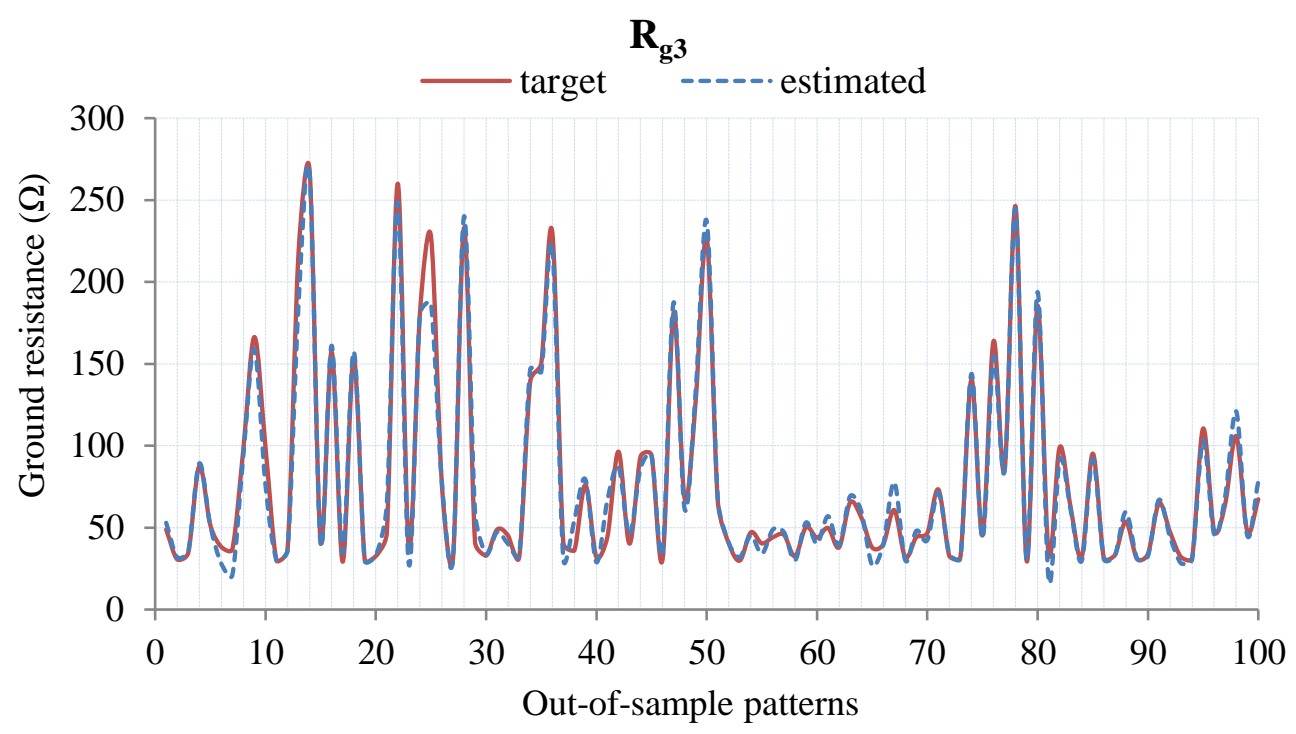

Fig. 8

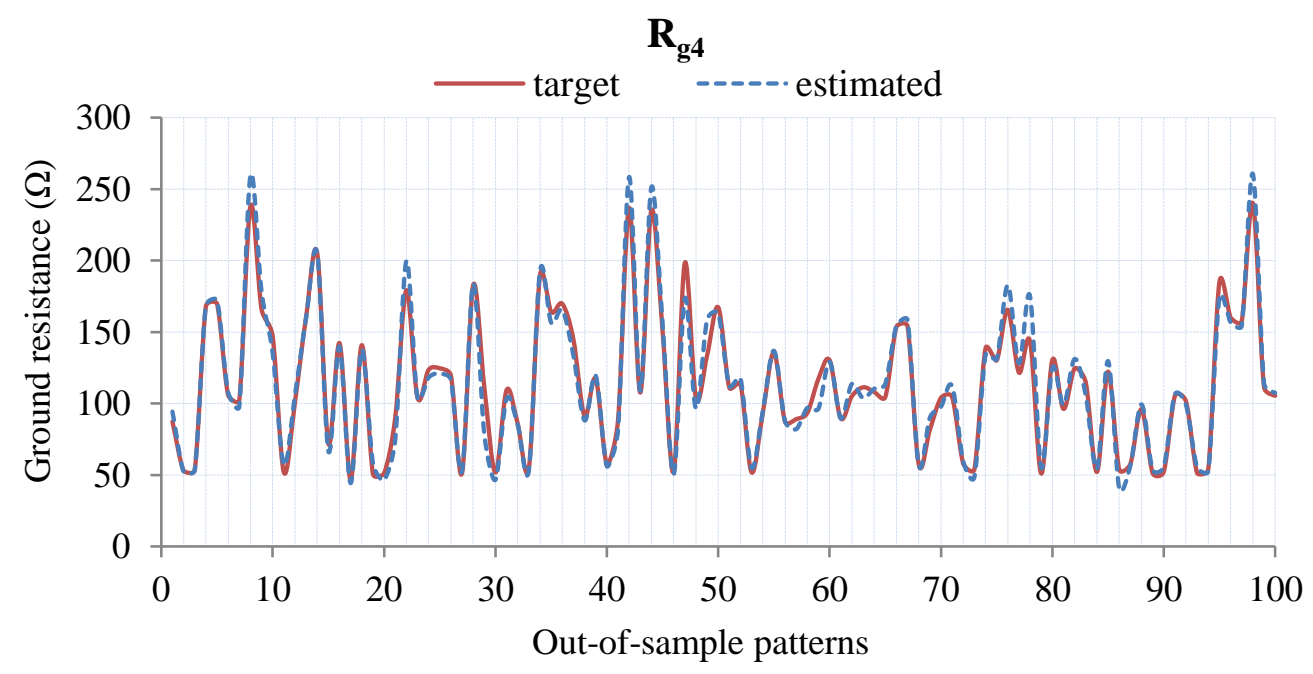

Fig. 9

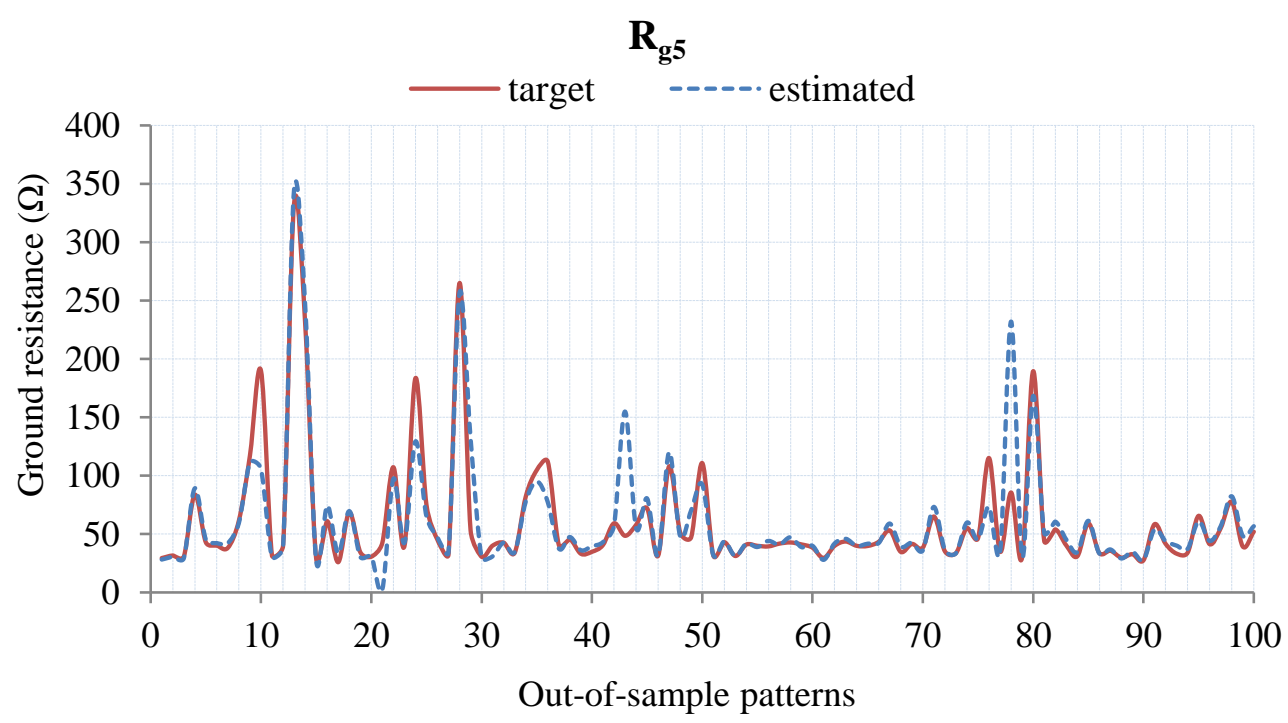

Table 1

Fig. 10 


\begin{tabular}{|c|c|c|c|}
\hline & & In-sample & Out-of-sample \\
\hline \multirow[t]{5}{*}{$\operatorname{Rg} 1$} & & \multicolumn{2}{|c|}{$\begin{array}{c}\text { Variables: } r_{d}, r_{w}, \rho_{1 d}, \rho_{4 d}, \rho_{1 w}, \rho_{6 w}, \rho_{8 w}, \rho_{1 m}, \rho_{2 m} \\
\text { HU: } 13\end{array}$} \\
\hline & NMSE & 0.0035 & 0.0768 \\
\hline & SMAPE \% & 1.17 & 4.04 \\
\hline & $\mathrm{R}^{2}$ & 0.9983 & 0.9644 \\
\hline & $\mathrm{R}^{2}$ adjusted & 0.9965 & 0.9232 \\
\hline \multirow[t]{5}{*}{$\operatorname{Rg} 2$} & & \multicolumn{2}{|c|}{$\begin{array}{c}\text { Variables: } \rho_{2 d}, \rho_{4 d}, \rho_{8 d}, \rho_{4 w}, \rho_{1 m}, \rho_{2 m} \\
H U: 16\end{array}$} \\
\hline & NMSE & 0.0188 & 0.0674 \\
\hline & SMAPE \% & 1.17 & 2.86 \\
\hline & & 0.9905 & 0.9665 \\
\hline & $\mathrm{R}^{2}$ adjusted & 0.9812 & 0.9326 \\
\hline \multirow[t]{5}{*}{$\operatorname{Rg} 3$} & & \multicolumn{2}{|c|}{$\begin{array}{c}\text { Variables: } r_{d}, r_{w}, r_{m}, \rho_{1 d}, \rho_{2 d}, \rho_{4 d}, \rho_{6 d}, \rho_{1 \mathrm{w}}, \rho_{6 \mathrm{w}}, \\
\rho_{8 \mathrm{w}}, \rho_{1 \mathrm{~m}} \quad \text { HU: } 12\end{array}$} \\
\hline & NMSE & 0.0037 & 0.0205 \\
\hline & SMAPE \% & 2.25 & 4.63 \\
\hline & & 0.9981 & 0.9898 \\
\hline & $\mathrm{R}^{2}$ adjusted & 0.9963 & 0.9795 \\
\hline \multirow[t]{5}{*}{$\operatorname{Rg} 4$} & & \multicolumn{2}{|c|}{$\begin{array}{c}\text { Variables: } \rho_{1 \mathrm{~d}}, \rho_{8 \mathrm{~d}}, \rho_{4 \mathrm{w}}, \rho_{1 \mathrm{~m}}, \rho_{2 \mathrm{~m}} \\
\text { HU: } 15\end{array}$} \\
\hline & NMSE & 0.0105 & 0.0371 \\
\hline & SMAPE \% & 1.33 & 2.79 \\
\hline & & 0.9948 & 0.9844 \\
\hline & $\mathrm{R}^{2}$ adjusted & 0.9895 & 0.9629 \\
\hline \multirow[t]{5}{*}{$\operatorname{Rg} 5$} & & \multicolumn{2}{|c|}{$\begin{array}{c}\text { Variables: } \rho_{4 \mathrm{~d}}, \rho_{6 \mathrm{~d}}, \rho_{8 \mathrm{~d}}, \rho_{2 \mathrm{w}}, \rho_{1 \mathrm{~m}} \\
\mathrm{HU}: 12\end{array}$} \\
\hline & NMSE & 0.0031 & 0.2536 \\
\hline & SMAPE \% & 2.35 & 7.57 \\
\hline & & 0.9985 & 0.8788 \\
\hline & $\mathrm{R}^{2}$ adjusted & 0.9969 & 0.7464 \\
\hline & NMSE: & \multirow{3}{*}{\multicolumn{2}{|c|}{$\begin{array}{l}\text { normalized mean squared error } \\
\text { symmetric mean absolute percentage error } \\
\text { coefficient of determination }\end{array}$}} \\
\hline & SMAPE: & & \\
\hline & $\mathrm{R}^{2}:$ & & \\
\hline
\end{tabular}

\section{Analysis}

The results show that employing the proposed WNN methodology, developed in this study, the estimation and forecast of ground resistance can be performed with great accuracy. Employing a WNN, a good fit on the data was obtained and the dynamics between the input and the output variables were discovered. This allowed the production of accurate out-of-sample forecast. In addition, it seems to constitute a useful and powerful tool for the disclosure of crucial data about the effect of rainfall 
and soil humidity on the behavior and the performance of ground enhancing compounds.

According to the results of Table 1, the SMAPE values of the WNN forecasts for the out-of-sample set lie on good levels, with the wavelet networks of the rods $\mathrm{G}_{2}$ (conductive concrete) and $\mathrm{G}_{4}$ (chemical compound $\mathrm{A}$ ) yielding the lowest values ( $2.86 \%$ and $2.79 \%$ respectively). This fact could be attributed to the more stable and, consequently, more predictable ground resistance these two grounding systems present throughout the forty four-month experiment (see Fig. 3). In addition, the $\mathrm{WN}$ for $\mathrm{G}_{3}$ (slurry bentonite) yields the best correlation results with the highest $R^{2}$ and adjusted $R^{2}$ coefficients, 0.9898 and 0.9795 respectively. This is also evident in Fig. 8 where, the almost real and forecasted values of the ground resistance are presented. The good performance of the WNN for the $\operatorname{rod} \mathrm{G}_{4}$ is also shown in Fig. 9, while Figs. 6-7 illustrate a slightly worse forecast (almost $2 \%$ lower) for the rods $\mathrm{G}_{1}$ and $\mathrm{G}_{2}$ than the corresponding one of $\mathrm{G}_{4}$.

On the contrary, for rod $\mathrm{G}_{5}$ the WNN produce slightly worse forecasts (Fig. 10). The values of $7.57 \%, 0.8788$ and 0.7464 for SMAPE, $R^{2}$ and adjusted $R^{2}$ are at a disadvantage against the corresponding values of the other materials despite the fact that, the chemical compound B presents the lowest ground resistance values for the biggest part of the experimental cycle.

The variable selection method has proved to be a valuable tool for the assessment and the characterization of each enhancing compound, based on the effect of rainfall and soil humidity on their behavior. More precisely, it is indicated that the rainfall variables are statistically significant for the prediction of ground resistance for the grounding systems $G_{1}$ and $G_{3}$ in contrast to the other systems. These results point out that the rainfall variables are too significant for the determination of the ground 
resistance for these two rods that cannot be removed. Hence, the soil in the testing field and the slurry bentonite are presented to be quite water-absorbent materials, whose resistance behavior is greatly affected by the presence of large amounts of moisture. Besides, this suggestion is verified by the graphs of Fig. 3 where one could observe the sharp fluctuation of ground resistance for the rods $G_{1}$ and $G_{3}$ in relation to rainfall height. Thus, the WNN methodology seems to be able to model the particular relationship ground resistance-rainfall for the assessment of various grounding systems. Additionally, the presence of each type of the rainfall variables, i.e. $r_{d}, r_{w}$ or $r_{m}$, can give valuable information about the: a) material "memory" (humidity-wise) and b) extent of the rainfall records affecting its attitude.

Furthermore, an overview of the input variables in Table 1 leads to the conclusion that the significance of each variable on ground resistance is strongly dependent on the nature of the tested material. For example, the soil resistivity values on the day of measurement in the deeper soil layers $\left(\rho_{4 d}, \rho_{6 \mathrm{~d}}, \rho_{8 \mathrm{~d}}\right)$ are more important parameters to $R_{g 5}$ than to $R_{g 4}$ which, in turn, is most affected by the mean monthly value of soil resistivity ( $\left.\rho_{i m}\right)$ of the upper layers. Of course, the absence of the rainfall variables from the input layer of the $\mathrm{WNN}$ for $\mathrm{G}_{2}, \mathrm{G}_{3}$ and $\mathrm{G}_{4}$ doesn't necessarily mean that there is no influence of rainfall on the resistance of these grounding systems. The rainfall parameters are simply less significant to the determination of ground resistance of these rods than to the others'; hence their effect is encompassed in soil resistivity variables. Our results indicate that the WNN methodology is also able to adapt to material composition and to estimate the ground resistance in a flexible and adaptive way.

\section{Conclusions}

A WNN based on back-propagation algorithm with batch training method and learning rate has developed, trained in order to forecast the variation of ground 
resistance. The present paper endeavors to construct WNN models applicable on ground resistance estimation, as an alternative to previous methodology developed in [17]. The developed WNN is a useful and reliable tool for assessing the performance and behavior of several ground enhancing compounds in grounding systems, which is the ultimate goal of this work. The presented results, in this study, show that the WNN was able to forecast with great accuracy the ground resistance for all rods.

IEC 60364-41 recommend the national standards to determine particular maximum values of ground resistance for building electrical installations. Thus, electrical engineers need to have a reliable and firm tool for the estimation of ground resistance during the whole year, since ground resistance variations may be significant with respect to time and rainfall (Fig. 3). This is very important, particularly, in the design of a grounding system. Therefore, the significance and the practical value of ground resistance estimation become indisputable and, from the results of this study, it seems that the proposed WNN methodology offers a valuable tool for this purpose.

The convergence results between the target and the estimated values as well the low SMAPE values show that the network performance is quite high. Moreover, the proposed WNN methodology is able to model the relationship among parameters such as rainfall, soil resistivity and compound composition, aiming the best ground resistance estimation.

Further work on variable selection methods could yield better results for the network. More particularly, a careful study of ground resistance in function of rainfall may lead to the determination of suitable time windows of rainfall as input vector to networks. Perhaps this will result in a simpler WNN architecture, smaller training times and more precise forecasts. 


\section{Appendix A}

For the best consideration and assessment of the training and forecasting sets, descriptive statistics of the in-sample and out-of-sample values are presented in Tables $\mathrm{A} 1$ and $\mathrm{A} 2$.

Table A1

\begin{tabular}{c|ccccccccccc} 
In-sample \\
\hline \hline Var & Mean & St.Dev & Max & Median & Min & Skewness & Kurtosis & KS & $p$-value & LBQ & $p$-value \\
\hline \hline $\mathrm{r}_{\mathrm{d}}$ & 0.57 & 3.02 & 37.80 & 0.00 & 0.00 & 9.34 & 105.13 & 7.70 & 0.0000 & 7.16 & 0.9961 \\
$\mathrm{r}_{\mathrm{w}}$ & 9.16 & 17.81 & 116.60 & 1.00 & 0.00 & 3.17 & 15.22 & 7.70 & 0.0000 & 28.29 & 0.1027 \\
$\mathrm{r}_{\mathrm{m}}$ & 42.50 & 44.03 & 188.00 & 29.20 & 0.00 & 1.31 & 4.08 & 12.87 & 0.0000 & 18.76 & 0.5376 \\
$\rho_{1 \mathrm{~d}}$ & 231.98 & 68.26 & 458.67 & 205.59 & 133.83 & 1.28 & 4.04 & 15.40 & 0.0000 & 12.06 & 0.9141 \\
$\rho_{2 \mathrm{~d}}$ & 181.11 & 44.30 & 326.47 & 170.40 & 126.67 & 0.97 & 3.27 & 15.40 & 0.0000 & 11.60 & 0.9291 \\
$\rho_{4 \mathrm{~d}}$ & 129.36 & 15.37 & 193.52 & 126.17 & 106.56 & 0.93 & 3.76 & 15.40 & 0.0000 & 14.41 & 0.8089 \\
$\rho_{6 \mathrm{~d}}$ & 154.51 & 14.54 & 267.66 & 153.06 & 134.81 & 2.36 & 17.21 & 15.40 & 0.0000 & 12.33 & 0.9043 \\
$\rho_{8 \mathrm{~d}}$ & 187.96 & 18.40 & 241.94 & 185.98 & 150.19 & 0.04 & 2.32 & 15.40 & 0.0000 & 28.67 & 0.0945 \\
$\rho_{1 \mathrm{w}}$ & 230.53 & 68.06 & 456.16 & 203.39 & 133.83 & 1.30 & 4.04 & 15.40 & 0.0000 & 10.81 & 0.9511 \\
$\rho_{2 \mathrm{w}}$ & 180.42 & 44.14 & 326.47 & 170.23 & 126.92 & 0.98 & 3.27 & 15.40 & 0.0000 & 11.30 & 0.9379 \\
$\rho_{4 \mathrm{w}}$ & 129.23 & 15.21 & 193.52 & 126.00 & 106.56 & 0.93 & 3.78 & 15.40 & 0.0000 & 15.06 & 0.7729 \\
$\rho_{6 \mathrm{w}}$ & 154.42 & 14.49 & 267.66 & 153.25 & 135.57 & 2.36 & 17.43 & 15.40 & 0.0000 & 12.57 & 0.8950 \\
$\rho_{8 \mathrm{w}}$ & 187.44 & 18.13 & 220.16 & 185.68 & 150.19 & -0.02 & 2.16 & 15.40 & 0.0000 & 24.39 & 0.2258 \\
$\rho_{1 \mathrm{~m}}$ & 225.60 & 64.25 & 425.76 & 196.46 & 157.21 & 1.37 & 4.01 & 15.40 & 0.0000 & 13.54 & 0.8532 \\
$\rho_{2 \mathrm{~m}}$ & 177.93 & 42.46 & 297.99 & 168.35 & 130.05 & 1.00 & 3.16 & 15.40 & 0.0000 & 13.85 & 0.8381 \\
\hline \hline $\mathrm{R}_{\mathrm{g} 1}$ & 268.30 & 127.14 & 676.00 & 235.00 & 93.60 & 0.75 & 3.01 & 15.40 & 0.0000 & 28.583 & 0.0963 \\
$\mathrm{R}_{\mathrm{g} 2}$ & 91.55 & 29.45 & 166.30 & 87.50 & 36.20 & -0.01 & 2.64 & 15.40 & 0.0000 & 18.883 & 0.5295 \\
$\mathrm{R}_{\mathrm{g} 3}$ & 83.82 & 61.16 & 281.00 & 60.80 & 29.10 & 1.49 & 4.39 & 15.40 & 0.0000 & 11.582 & 0.9297 \\
$\mathrm{R}_{\mathrm{g} 4}$ & 122.65 & 47.04 & 276.60 & 118.70 & 46.90 & 0.4 & 2.85 & 15.40 & 0.0000 & 28.759 & 0.0926 \\
$\mathrm{R}_{\mathrm{g} 5}$ & 74.93 & 72.80 & 376.00 & 44.60 & 26.20 & 2.5 & 8.56 & 15.40 & 0.0000 & 11.987 & 0.9165
\end{tabular}

Table A2

Out-of-sample

\begin{tabular}{c|ccccccccccc}
\hline \hline Var & Mean & St.Dev & Max & Median & Min & Skewness & Kurtosis & KS & $p$-value & LBQ & $p$-value \\
\hline \hline $\mathrm{r}_{\mathrm{d}}$ & 0.48 & 1.42 & 8.20 & 0.00 & 0.00 & 3.78 & 17.12 & 5.00 & 0.0000 & 15.00 & 0.7762 \\
$\mathrm{r}_{\mathrm{w}}$ & 9.37 & 16.90 & 91.80 & 1.40 & 0.00 & 2.81 & 11.62 & 5.00 & 0.0000 & 22.96 & 0.2906 \\
$\mathrm{r}_{\mathrm{m}}$ & 46.33 & 44.76 & 188.00 & 35.40 & 0.00 & 1.25 & 4.11 & 8.67 & 0.0000 & 26.04 & 0.1645 \\
$\rho_{1 \mathrm{~d}}$ & 220.12 & 63.38 & 447.36 & 191.89 & 156.33 & 1.56 & 4.96 & 10.00 & 0.0000 & 17.96 & 0.5901 \\
$\rho_{2 \mathrm{~d}}$ & 173.82 & 42.35 & 306.62 & 163.62 & 127.17 & 1.09 & 3.57 & 10.00 & 0.0000 & 18.08 & 0.5821 \\
$\rho_{4 \mathrm{~d}}$ & 127.85 & 13.75 & 165.88 & 124.41 & 112.09 & 0.76 & 2.67 & 10.00 & 0.0000 & 17.74 & 0.6045
\end{tabular}




\begin{tabular}{l|ccccccccccc}
$\rho_{6 \mathrm{~d}}$ & 153.07 & 15.23 & 248.06 & 151.89 & 135.26 & 2.54 & 16.20 & 10.00 & 0.0000 & 20.68 & 0.4165 \\
$\rho_{8 \mathrm{~d}}$ & 185.19 & 18.84 & 220.16 & 184.12 & 151.20 & 0.15 & 1.85 & 10.00 & 0.0000 & 17.70 & 0.6075 \\
$\rho_{1 \mathrm{w}}$ & 219.51 & 63.23 & 448.62 & 192.06 & 156.33 & 1.57 & 5.07 & 10.00 & 0.0000 & 18.82 & 0.5335 \\
$\rho_{2 \mathrm{w}}$ & 173.42 & 41.97 & 310.39 & 164.53 & 127.49 & 1.13 & 3.74 & 10.00 & 0.0000 & 21.16 & 0.3881 \\
$\rho_{4 \mathrm{w}}$ & 127.87 & 13.92 & 168.39 & 123.74 & 111.92 & 0.79 & 2.73 & 10.00 & 0.0000 & 18.54 & 0.5521 \\
$\rho_{6 \mathrm{w}}$ & 153.03 & 15.27 & 248.06 & 150.77 & 135.98 & 2.52 & 16.07 & 10.00 & 0.0000 & 20.65 & 0.4181 \\
$\rho_{8 \mathrm{w}}$ & 184.68 & 18.78 & 220.16 & 183.90 & 151.15 & 0.17 & 1.88 & 10.00 & 0.0000 & 18.20 & 0.5741 \\
$\rho_{1 \mathrm{~m}}$ & 215.95 & 59.13 & 420.00 & 191.27 & 157.72 & 1.55 & 4.76 & 10.00 & 0.0000 & 21.95 & 0.3432 \\
$\rho_{2 \mathrm{~m}}$ & 171.52 & 41.02 & 293.45 & 163.03 & 130.06 & 1.06 & 3.31 & 10.00 & 0.0000 & 25.98 & 0.1666 \\
\hline \hline $\mathrm{R}_{\mathrm{g} 1}$ & 242.32 & 125.10 & 527.00 & 198.25 & 92.40 & 0.76 & 2.26 & 10.00 & 0.0000 & 2.80 & 0.8859 \\
$\mathrm{R}_{\mathrm{g} 2}$ & 85.08 & 31.48 & 161.40 & 84.50 & 34.00 & 0.03 & 2.22 & 10.00 & 0.0000 & 10.51 & 0.9580 \\
$\mathrm{R}_{\mathrm{g} 3}$ & 77.60 & 62.23 & 268.00 & 47.65 & 29.20 & 1.58 & 4.44 & 10.00 & 0.0000 & 16.82 & 0.6646 \\
$\mathrm{R}_{\mathrm{g} 4}$ & 113.91 & 48.26 & 240.00 & 107.90 & 46.50 & 0.59 & 2.94 & 10.00 & 0.0000 & 15.53 & 0.7452 \\
$\mathrm{R}_{\mathrm{g} 5}$ & 59.92 & 50.38 & 332.90 & 41.60 & 25.90 & 3.2 & 14.33 & 10.00 & 0.0000 & 26.20 & 0.1592
\end{tabular}

where

- KS: Kolmogorov Smirnof distance is a measure of the distance between the data distribution and the normal distribution.

- LBQ: Ljung-Box Q statistic is a measure of the possible autocorrelation among the data.

- p-value: the p-value for each of the descriptive statistics KS and LBQ

\section{References}

[1] IEEE Std 142-2007, "IEEE Recommended Practice of Grounding for Industrial and Commercial Power Systems”, IEEE Green Book, Nov. 30 ${ }^{\text {th }}, 2007$.

[2] ANSI/IEEE Std 80-2013, "IEEE Guide for Safety in AC Substation Grounding", Apr. 2013.

[3] ANSI/IEEE Std 81-2012, "IEEE guide for measuring earth resistivity, ground impedance and earth surface potentials of a grounding system”, Dec. $28^{\text {th }}, 2012$.

[4] I.F. Gonos, A.X. Moronis, I.A. Stathopulos, Variation of soil resistivity and ground resistance during the year, in: Proc. of $28^{\text {th }}$ Int. Conf. Lightning Protection 
(ICLP 2006), Kanazawa, Japan, September $18^{\text {th }}-22^{\text {nd }}, \quad 2006$, pp. 740-746.

[5] O. Banton, M.A. Cimon, M.K. Seguin, Mapping field-scale physical properties of soil with electrical resistivity, Soil Sci. Soc. of America J. 61 (4) (1997) 1010-1017.

[6] K. Shudha, M. Israil, S. Mittal, J. Rai, Soil characterization using electrical resistivity tomography and geotechnical investigations, J. Appl. Geophys. 67 (January (1)) (2009) 74-79.

[7] L. Cao, Y. Hong, H. Fang, G. He, Predicting chaotic time series with wavelet networks, Physica D: Nonlinear Phenomena 85 (July (1-2)) (1995) 225-238.

[8] Y. Fang, T.W.S. Chow, Wavelets based neural network for function approximation, Lect. Notes Comput. Sci. 3971 (2006) 80-85.

[9] Q. Zhang, Using wavelet network in nonparametric estimation, IEEE Trans. Neural Netw. 8 (March (2)) (1997) 227-236.

[10] A.K. Alexandridis, A.D. Zapranis, Wavelet neural networks: A practical guide, Neural Netw. 42 (June) (2013) 1-27.

[11] A.K. Alexandridis, A.D. Zapranis, Wavelet Neural Networks: With Applications in Financial Engineering, Chaos and Classification, First Edition, John Wiley \& Sons, Inc., Hoboken, New Jersey, 2014.

[12] D.L. Donoho, J.M. Johnstone, Ideal spatial adaption by wavelet shrinkage, Biometrica 81 (September (3)) (1994) 425-455.

[13] J.B. Ramsey, The contribution of wavelets to the analysis of economic and financial data, Philosph. Trans.: Math., Phys. and Eng. Sci. 357 (September (1760)) (1999) 2593-2606. 
[14] Q. Zhang, A. Benveniste, Wavelet networks, IEEE Trans. Neural Netw. 3 (November (6)) (1992) 889-898.

[15] C. Bernard, S. Mallat, J.-J. Slotine, Wavelet interpolation networks, in: Proc. of $6^{\text {th }}$ Europ. Symp. Artif. Neural Netw. (ESANN '98), Bruges, Belgium, April 22 $2^{\text {nd }}$ $24^{\text {th }}, 1998$, pp. $47-52$.

[16] V.P. Androvitsaneas, I.F. Gonos, I.A. Stathopulos, Performance of ground enhancing compounds during the year, in: Proc. of $31^{\text {st }}$ Int. Conf. Lightning Protection (ICLP 2012), Vienna, Austria, September $2^{\text {nd }}-7^{\text {th }}, 2012$.

[17] V.P. Androvitsaneas, I.F. Gonos, I.A. Stathopulos, Artificial neural network methodology for the estimation of ground enhancing compounds resistance, IET Sci. Meas. Technol. 8 (November (6)) (2014) 552-570.

[18] Y. Becerikli, Y. Oysal, A.F. Konar, On a dynamic wavelet network and its modeling application, Lect. Notes Comput. Sci. 2714 (2003) 710-718.

[19] S.A. Billings, H.-L. Wei, A new class of wavelet networks for nonlinear system identification, IEEE Trans. Neural Netw. 16 (July (4)) (2005) 862-874.

[20] Q. Zhang, Using wavelet network in nonparametric estimation, 2321 Technical Report INRIA 1994.

[21] V.P. Androvitsaneas, A.K. Alexandridis, I.F. Gonos, G.D. Dounias, I.A. Stathopulos, Wavelet neural network for ground resistance estimation, in: Proc. of $4^{\text {th }}$ Int. Conf. High Voltage Eng. Appl. (ICHVE 2014), Poznan, Poland, September $8^{\text {th }}-11^{\text {th }}, 2014$.

\section{Table and Figure Captions}

Table 1. Results of the WNN performance for the in-sample and out-of-sample sets.

Fig. 1. Structure of a feedforward Wavelet Neural Network [21].

Fig. 2. Flowchart of the proposed WNN methodology [21]. 
Fig. 3. Schematic representation of a grounding rod encased in ground enhancing compound.

Fig. 4. Soil resistivity as a function of time and rainfall.

Fig. 5. Ground resistance of the tested grounding rods as a function of time and rainfall.

Fig. 6. Target and estimated values of ground resistance for grounding system $\mathrm{G}_{1}$.

Fig. 7. Target and estimated values of ground resistance for grounding system $\mathrm{G}_{2}$.

Fig. 8. Target and estimated values of ground resistance for grounding system $\mathrm{G}_{3}$.

Fig. 9. Target and estimated values of ground resistance for grounding system $\mathrm{G}_{4}$.

Fig. 10. Target and estimated values of ground resistance for grounding system $\mathrm{G}_{5}$. 